\title{
An audit of antimicrobial prescribing by dental practitioners in the north east of England and Cumbria
}

\author{
A. Sturrock ${ }^{1 *}$ D, D. Landes ${ }^{2}$, T. Robson ${ }^{3}$, L. Bird ${ }^{4}$, A. Ojelabi ${ }^{1}$ and J. Ling ${ }^{1}$
}

\begin{abstract}
Background: Inappropriate prescribing of antimicrobials is a significant threat to global public health. In England, approximately $5 \%$ of all antimicrobial items are prescribed by dentists, despite the limited indications for their use in the treatment of oral infections in published clinical guidelines. The objective of this study was to survey antimicrobial prescribing by dental practitioners in North East England and Cumbria, identify educational and training needs and develop a self-assessment tool that can be used for Continued Professional Development by individual practitioners.
\end{abstract}

Methods: During October 2016, 275 dental practitioners used a standardised form to record anonymous information about patients who had been prescribed antimicrobials. Clinical information and prescribing details were compared against clinical guidelines published by the Faculty of General Dental Practitioners UK.

Results: Dental practitioners provided data on 1893 antimicrobial prescriptions. There was documented evidence of systemic spread, such as pyrexia in 18\% of patients. Dentists recorded patients' pain ( $91.1 \%$ of patients), local lymph gland involvement (41.5\%) gross diffuse swelling (55.5\%) dysphagia (7.2\%) and trismus (13.6\%). Reasons for prescribing antimicrobials included patient expectations (25.8\%), patient preference (24.8\%), time pressures (10.9\%), and patients uncooperative with other treatments (10.4\%). The most commonly prescribed antimicrobials were amoxicillin, accounting for $61.2 \%$ of prescriptions, followed by metronidazole (29.9\%). Most prescriptions for amoxicillin were for either 5 days (66.8\%) or 7 days (29.6\%) and most prescriptions for metronidazole were for a 5 day course $(65.2 \%)$ or 7 -day (18.6\%) course.

Conclusion: In most cases, when an antimicrobial was prescribed, practitioners used the correct choice of agents and usually prescribed these at the correct dose. However, some evidence of suboptimal prescribing practices when compared to the Faculty of General Dental Practitioner guidelines were identified. The audit has identified training needs across the region and aided the development of Continued Professional Development sessions. Further work to identify barriers and facilitators for improving antimicrobial prescribing and determining appropriate methods to improve clinical practice are required.

Keywords: Antibiotics, Audit, Dental, Prescribing

\footnotetext{
* Correspondence: andrew.sturrock@sunderland.ac.uk

${ }^{1}$ Faculty of Health Sciences and Wellbeing, Sciences Complex, City Campus,

Chester Road, University of Sunderland, Sunderland SR1 3SD, UK

Full list of author information is available at the end of the article
}

(c) The Author(s). 2018 Open Access This article is distributed under the terms of the Creative Commons Attribution 4.0 International License (http://creativecommons.org/licenses/by/4.0/), which permits unrestricted use, distribution, and reproduction in any medium, provided you give appropriate credit to the original author(s) and the source, provide a link to the Creative Commons license, and indicate if changes were made. The Creative Commons Public Domain Dedication waiver (http://creativecommons.org/publicdomain/zero/1.0/) applies to the data made available in this article, unless otherwise stated. 


\section{Background}

Antimicrobial resistance is a significant threat to global public health. The World Health Organisation has stressed the seriousness of this problem and their Global Report of Surveillance in 2014 (p. IX) concluded that 'A post-antimicrobial-era in which common infections and minor injuries can kill - is a very real possibility for the $21^{\text {st }}$ century' [1].

Antimicrobial stewardship is defined by the UK National Institute for Health and Care Excellence (NICE) as 'an organisational or healthcare-system-wide approach to promoting and monitoring judicious use of antimicrobials to preserve their future effectiveness' [2]. Therefore commissioners, providers and individual practitioners should embrace antimicrobial stewardship with the view to improving practice [2]. When prescribing antimicrobials, prescribers should follow local antimicrobial guidelines, where available, or national guidelines, such as the UK Faculty of General Dental Practitioners (FGDP) guidelines on prescribing the shortest effective course, the most appropriate dose and the route of administration for appropriate clinical indications [3].

The FGDP antimicrobial prescribing guidelines for general dental practitioners recommends that antimicrobials are only indicated for the following [3]:

- As an adjunct to the management of acute or chronic infection

- For the definitive management of active infective disease such as necrotising ulcerative gingivitis

- Where definitive treatment has to be delayed due to referral to a specialist service, examples include inability to establish drainage in an uncooperative patient who requires sedation or general anaesthesia for treatment, or a patient who needs to be treated in a hospital environment due to comorbidities.

Antibiotic consumption has increased by $6.5 \%$ over the past 4 years in England [4]. Prescribing is measured as the defined daily dose (DDD) of antimicrobials taken by an individual per 1000 inhabitants per day. Prescribing increased from 21.6 DDD per 1000 inhabitants per day in England in 2011 to 23 DDD per 1000 inhabitants per day in 2014 [4]. In 2014, the majority (66.6\%) of dental prescriptions were used to treat infections and although dental prescribing accounts for only $0.5 \%$ of all items prescribed in England [4], dentists are responsible for $5 \%$ of all antimicrobial drug prescriptions [5]. The highest combined general practice, hospital and dentist usage in England in 2015 at 27.4 DDD per 1000 inhabitants per day was Merseyside. Antibiotic prescribing is also high in the North East of England, with the second highest DDD nationally (25.8) in Cumbria, Northumberland and Tyne \& Wear and the third highest (25.3) in
Durham, Darlington and Tees [5]. Antibiotic consumption was $26.5 \%$ higher in Cumbria, Northumberland and Tyne and Wear than in Leicestershire and Lincolnshire which had the lowest usage of antimicrobials at 20.4 DDD per 1000 inhabitants per day [5]. The potential reasons for the variations in regional prescribing practices are unclear, this may be due to socioeconomic factors, although studies have found that this can only partly explain differences in antimicrobial use [6].

Clinical audit can provide practitioners with a way of assessing their compliance with current prescribing guidelines and there is evidence that antimicrobial prescribing can be improved following clinical audit, leading to a reduction in inappropriate prescribing and prescribing errors [7-9]. A recent audit of dental antimicrobial prescribing in Wales provides a benchmark for assessing prescribing in the North East and Cumbria. The authors found that $21.8 \%$ of antimicrobial prescriptions did not meet the dose, frequency or duration advised in clinical guidelines [9]. The specific prescribing choices for each indication were not reported; therefore our study has been designed to further explore the antimicrobial choices for each clinical diagnosis and present detailed prescribing information to further support the literature in this field.

The aim of this study was to produce data on antimicrobial prescribing by dental practitioners in the North East of England and Cumbria.

\section{Methods \\ Design}

The study was designed to capture prospective data on antimicrobial prescribing by dental practitioners in the North East and Cumbria during the month of October 2016. Dentists were asked to record information on the next 10 consecutive antimicrobial prescriptions they gave to patients from the start of the census period. The purpose of the audit tool was to capture the prescribing practices of participants and not the frequency of prescribing The standard used in the audit was the Faculty of General Dental Practitioners antimicrobial guidelines [3]. Comparative data for dental prescriptions dispensed by pharmacies in the North East of England and Cumbria during the month of the audit were also provided by the NHS Business Service Authority (BSA).

\section{Population}

The population consisted of all dentists (2318) on the National Health Service (NHS) performer list working under NHS contracts in the North East and Cumbria. Participants were identified via NHS England, who posted all study materials to the registered address of providers with a general dental services or community dental services contract in the North East and Cumbria. A reminder letter was sent half way through the audit period. 


\section{Audit tool}

The audit tool (see Additional file 1) consisted of two parts. The first section captured demographic information of participating dental practitioners. This information included gender, age, country of undergraduate study and area of current practice. Information regarding the size of their practice, Dental Foundation Training or General Professional Training practice status and the number of patients typically seen per day was also obtained. The audit tool was piloted in two practices before being finalised.

The second section of the audit tool captured information on 10 antimicrobial prescriptions. Participants were asked to record information on the next 10 consecutive antimicrobial prescriptions they gave to patients; if participants did not prescribe antimicrobials to 10 patients during the audit period (October 2016), they were asked to return the audit tool with the data from the patients for whom they did prescribe.

The following information was recorded for each antimicrobial prescription:

- Patient information - age and allergy status

- Diagnosis and features - clinical diagnosis and presenting features, such as pyrexia, pain, local lymph gland involvement, diffuse swelling, dysphagia, trismus

- Antibiotic prescribed - name, dose, quantity, frequency, duration

- Influencing factors - patient expectations, preferences, time pressures, patients condition, uncertain diagnosis, failure of anaesthesia, uncooperative patients and failure of other treatments

\section{Procedure}

The audit tool was posted by NHS England to dental practices in the North East and Cumbria during September 2016. A covering letter was included, explaining the background and purpose of the audit, the letter explained that the audit was voluntary, but would provide participants with $3 \mathrm{~h}$ of Continued Professional Development (CPD) for completing and returning the audit. A reminder letter was sent to all providers 2 weeks into the audit period.

\section{Ethics}

The project was approved by the University of Sunderland Research Ethics Committee.

\section{Analysis}

Completed audit templates were returned to the University of Sunderland for data entry and analysis using SPSS.

\section{Results}

Based on data received from NHS England, there are an estimated 2318 performers in the North East and Cumbria. A total of 275 dental practitioners returned data on the audit, with a response rate of $11.9 \%$. Practitioners provided data on 1893 antimicrobial prescriptions.

\section{Participant demographics}

Of the 275 participants, 56\% were male and $37.1 \%$ female; the remainder did not state their gender. The vast majority $(81.8 \%)$ of participants had undertaken their undergraduate dental training in the UK, with the remaining studying in Europe (7.3\%), and outside of Europe $(4 \%) ; 6.9 \%$ of participants did not provide information on where they studied. Most participants described themselves as general dental practitioners (88.4\%) with several orthodontists (2.2\%) and community dentists $(0.7 \%)$, also participating. The area of practice was missing from $8 \%$ of audit returns.

The largest proportion of responses came from Tyne and Wear with $35.6 \%$, with Northumbria (10.2\%), showing the smallest return. These returns are broadly as expected given the relative sizes of the populations of each of these areas. Data were missing from $6.9 \%$ of participants and the region was uncertain from the responses in $0.7 \%$ of participants.

Participants were asked to provide information on the practice in which they worked, $32.2 \%$ stated they worked in a Vocational Training (VT) or General Professional Training (GPT) practice; both VT and GPT practices are approved sites for the post-qualification training period required for UK graduates in order to work in NHS practice [10]. The modal number of practitioners working at a practice was 3 (range 1-23). The majority of participants, $62.5 \%$, reported that they saw $21-30$ patients per day.

\section{Antibiotic prescribing}

Data on a total of 1893 antimicrobial prescriptions were reported in the audit. The mean age of patients who were prescribed antimicrobials was 43.02 years (SD 19.67 years); patients' ages ranged from 1 to 91 years.

Allergy information was recorded for the majority of patients (see Table 1); this information was missing for 92 patients. Most patients (85.1\%) did not have a known antimicrobial allergy; the most frequent antimicrobial allergy reported by patients was for amoxicillin, for $7.3 \%$ of patients.

Antibiotics were prescribed for a range of indications. The most common indication was an acute dento-alveolar infection (43.2\% of prescriptions), followed by pericoronitis (14.7\%) and periodontal abscess $(11.7 \%)$. The indication was described as 'other' in $3 \%$ of prescriptions with no further description given. Indication was missing from the remaining $2.9 \%$ of prescriptions (see Table 2). 
Table 1 Frequency of documented antibiotic allergies

\begin{tabular}{lll}
\hline Antibiotic & Frequency & Percent \\
\hline Amoxicillin & 135 & 7.1 \\
Metronidazole & 19 & 1 \\
Phenoxymethylpenicillin & 10 & 0.5 \\
Erythromycin & 6 & 0.3 \\
Amoxicillin and Phenoxymethylpenicillin & 3 & 0.2 \\
Cefalexin & 3 & 0.2 \\
Amoxicillin and Erythromycin & 2 & 0.1 \\
Amoxicillin and Metronidazole & 2 & 0.1 \\
Amoxicillin and Ampicillin & 2 & 0.1 \\
Amoxicillin, Ampicillin, Phenoxymethylpenicillin & 2 & 0.1 \\
Ampicillin & 2 & 0.1 \\
Amoxicillin, Ampicillin, Phenoxymethylpenicillin, & 1 & 0.1 \\
Co-amoxiclav & & \\
Phenoxymethylpenicillin and Metronidazole & 1 & 0.1 \\
Azithromycin & 1 & 0.1 \\
Clindamycin & 1 & 0.1 \\
No known antibiotic allergy & 1611 & 85.1 \\
No information provided & 92 & 4.9 \\
Total & 1893 & 100 \\
\hline
\end{tabular}

There was documented evidence of systemic spread, such as pyrexia in $18 \%$ of patients. Pain was reported in $91.1 \%$ of patients. Local lymph gland involvement was evident in $41.5 \%$ of prescriptions, with gross diffuse swelling present in $55.5 \%$ of cases. Dysphagia and trismus was present in 7.2 and $13.6 \%$ of patients respectively.

The most commonly prescribed antimicrobials were amoxicillin, accounting for $61.2 \%$ of prescriptions, followed by metronidazole (29.9\%). The vast majority of patients were prescribed only one antimicrobial. Where multiple antimicrobials were prescribed, the most frequent combination was amoxicillin and metronidazole (1.7\%). The frequency of each antimicrobial prescribed is shown in Table 3.

Data for dental prescriptions dispensed by pharmacies in the North East of England and Cumbria during the month of the audit was provided by the NHS Business Service Authority (BSA) (Additional file 2). The number of prescriptions issued for each antimicrobial is shown in Table 4. The frequency of prescriptions for each antimicrobial closely matches the data from this audit.

\section{Amoxicillin}

Amoxicillin was typically prescribed at a dose of $500 \mathrm{mg}$ (78.5\%) or $250 \mathrm{mg}(16.8 \%)$ and usually three times daily (98.3\%). Most prescriptions for amoxicillin were for either 5 days $(66.8 \%)$ or 7 days $(29.6 \%)$ duration; the mean duration was 5.61 days (SD 1.06 days).
Table 2 Frequency of reported clinical diagnosis

\begin{tabular}{lll}
\hline Clinical Diagnosis & Frequency & Percent \\
\hline Acute dento-alveolar infection & 817 & 43.2 \\
Pericoronitis & 279 & 14.7 \\
Periodontal abscess & 221 & 11.7 \\
Dry socket & 145 & 7.7 \\
Chronic dento-alveolar infection & 130 & 6.9 \\
Acute Dental Pain/Pulpitis & 62 & 3.3 \\
Necrotising ulcerative gingivitis & 58 & 3.1 \\
Other & 57 & 3 \\
Prophylactic antibiotics & 25 & 1.3 \\
Acute sinusitis & 13 & 0.7 \\
Gingivitis & 12 & 0.6 \\
Acute dento-alveolar infection and Acute & 2 & 0.1 \\
Dental Pain/Pulpitis & & \\
Acute dento-alveolar infection and Chronic & 2 & 0.1 \\
dento-alveolar infection & & \\
Acute dento-alveolar infection and & 2 & 0.1 \\
Periodontal abscess & 1893 \\
Acute dento-alveolar infection and Dry socket & 2 & 0.1 \\
Periodontal abscess and Necrotising & 2 & 0.1 \\
ulcerative gingivitis & 1 & 0.1 \\
Acute dento-alveolar infection and Gingivitis & 1 & 0.1 \\
Acute dento-alveolar infection and Acute sinusitis & 1 & 0.1 \\
Necrotising ulcerative gingivitis and Dry socket & 1 & 0.1 \\
Pericoronitis and others & 1 & 0.1 \\
Local anaesthetic complication & 1 & 0.1 \\
Stomatitis & 1 & \\
Tonsillitis & 1 & \\
Trauma & 1 & \\
No information provided & 1 & \\
Total & 1 & 0.1 \\
\hline
\end{tabular}

\section{Metronidazole}

Metronidazole was the second most frequent antimicrobial prescribed, with doses of $400 \mathrm{mg}$ accounting for $53.8 \%$ and $200 \mathrm{mg}, 42.6 \%$ of cases. Almost all (98.1\%) of prescriptions were for three times daily dosing and the mean duration of course was 5.13 days (SD 1.14). Most prescriptions were for a 5-day course (65.2\%), 7-day (18.6\%) or 3-day (12.9\%) course.

\section{Erythromycin}

Erythromycin was the third most prescribed antimicrobial identified in the audit (2.8\%), when prescribed it was usually given at a dose of $250 \mathrm{mg}(60.4 \%)$ or $500 \mathrm{mg}$ (32.1\%). It was prescribed 3 times daily in $22.6 \%$ or 4 times daily in $75.5 \%$ of cases. Erythromycin was prescribed for a mean duration of 5.49 days (SD 1.01). 
Table 3 Frequency of each antimicrobial prescribed

\begin{tabular}{lll}
\hline Antibiotic & Frequency & Percent \\
\hline Amoxicillin & 1159 & 61.2 \\
Metronidazole & 566 & 29.9 \\
Erythromycin & 53 & 2.8 \\
Amoxicillin and Metronidazole & 33 & 1.7 \\
Cefalexin & 8 & 0.4 \\
Clarithromycin & 7 & 0.4 \\
Clindamycin & 5 & 0.3 \\
Co-amoxiclav & 5 & 0.3 \\
Other (specify) & 4 & 0.2 \\
Phenoxymethylpenicillin & 3 & 0.2 \\
Ampicillin & 2 & 0.1 \\
Cefradine & 2 & 0.1 \\
Doxycycline & 2 & 0.1 \\
Azithromycin & 1 & 0.1 \\
Clindamycin and Metronidazole & 1 & 0.1 \\
Co-amoxiclav and Metronidazole & 1 & 0.1 \\
No information provided & 41 & 2.2 \\
Total & 1893 & 100 \\
\hline
\end{tabular}

\section{Reasons for prescribing antimicrobials}

The reasons for prescribing an antimicrobial was documented for $91.9 \%$ of patients (participants could document more than one reason if appropriate). Data were missing for $8.1 \%$ of patients. Table 5 illustrates the reasons for prescribing and the frequency of responses. The patient's condition was the most frequent reason given

Table 4 The number of dental antibiotic prescriptions issued by pharmacies in the North East of England and Cumbria (NHS BSA prescribing data October 2016)

\begin{tabular}{lll}
\hline Antibiotic & $\begin{array}{l}\text { Number of } \\
\text { Prescriptions }\end{array}$ & $\begin{array}{l}\text { Percent of } \\
\text { prescriptions }\end{array}$ \\
\hline Amoxicillin & 9336 & 68.82 \\
Metronidazole & 3631 & 26.77 \\
Erythromycin & 420 & 3.10 \\
Phenoxymethylpenicillin & 44 & 0.32 \\
Co-amoxiclav & 33 & 0.24 \\
Clarithromycin & 30 & 0.22 \\
Clindamycin & 23 & 0.17 \\
Cefalexin & 21 & 0.15 \\
Doxycycline & 14 & 0.10 \\
Tetracycline & 8 & 0.06 \\
Oxytetracycline & 3 & 0.02 \\
Azithromycin & 2 & 0.01 \\
Total & 13,565 & 100.00 \\
\hline
\end{tabular}

Table 5 Reported reason for issuing antimicrobial prescription

\begin{tabular}{llll}
\hline Reason for prescribing & \multicolumn{2}{l}{ Responses } & $\begin{array}{l}\text { Percent } \\
\text { of Cases }\end{array}$ \\
\cline { 2 - 3 } & Number & Percent nn & \\
\hline Patient's condition & 1572 & $50.8 \%$ & $90.4 \%$ \\
Patient expectations & 448 & $14.5 \%$ & $25.8 \%$ \\
Patient preference & 432 & $13.9 \%$ & $24.8 \%$ \\
Time pressures & 190 & $6.1 \%$ & $10.9 \%$ \\
Patient uncooperative with & 181 & $5.8 \%$ & $10.4 \%$ \\
Other treatment & & & \\
Unclear/uncertain diagnosis & 116 & $3.7 \%$ & $6.7 \%$ \\
Failure of other treatment & 111 & $3.6 \%$ & $6.4 \%$ \\
Failure of anaesthesia & 47 & $1.5 \%$ & $2.7 \%$ \\
Total & 3097 & $100 \%$ & $178.1 \%$ \\
\hline
\end{tabular}

for prescribing an antimicrobial and was a documented factor in $90.4 \%$ of cases. This was followed by patient expectations and patient preference, 25.8 and $24.8 \%$ of cases respectively. Time pressure (10.9\%), and patients uncooperative with other treatment $(10.4 \%)$, were also reported by participants.

\section{Acute dento-alveolar infections}

According to the FGDP guidelines, the first choice treatment for this indication is amoxicillin which should be prescribed for adults and children over the age of 6 years at a dose of $500 \mathrm{mg} 3$ times daily for up to 5 days. We found that amoxicillin was the most frequently prescribed antimicrobial and that the duration of treatment was in most cases 5 days (68\%), although a number of prescriptions (28.6\%) were for 7 days treatment. A lower dose of $250 \mathrm{mg}$ was prescribed in $13.9 \%$ of cases. Systemic spread, such a pyrexia (18\%) or local lymph glad involvement (41.5\%), were not always present when prescribing antimicrobials.

Metronidazole is the second choice agent for this indication and in patients over 10 years of age should be prescribed at a dose of $400 \mathrm{mg}$ three times daily for up to 5 days. The duration of prescribing matched the guidelines in $68.5 \%$ of cases, but prescriptions for 7 days duration $(20.7 \%)$ were relatively frequent.

\section{Periodontal abscess}

Treatment recommendations for a periodontal abscess are the same as those for an acute dento-alveolar infection. The most frequently prescribed antimicrobial for this indication was amoxicillin, typically prescribed for 5 days $(61.5 \%)$ duration, or 7 days, (37.6\%). Where metronidazole was prescribed, it was usually for 5 days $(68.5 \%)$, or 7 days $(20.7 \%)$. In $10 \%$ of cases amoxicillin was prescribed at a lower dose of $250 \mathrm{mg}$. 


\section{Pericoronitis}

FGDP guidelines recommend that treatment of pericoronitis should follow local measures unless patients present with pyrexia, spreading infection, persistent swelling or trismus. Where an antimicrobial is recommended, metronidazole should be the first choice, at a dose of $400 \mathrm{mg}$ three times daily (patients aged over 10 years) for up to 5 days. The most frequently prescribed antimicrobial for the treatment of pericoronitis was metronidazole, typically prescribed for 5 days, (69.8\%) duration or 3 days (16.4\%). Where amoxicillin was prescribed, it was usually for 5 days $(61.8 \%)$ or 7 days $(35.5 \%)$ duration.

\section{Chronic dento-alveolar infection}

Where antimicrobial treatment is required the treatment should follow the guidance for the treatment of acute dento-alveolar infections. The most frequently prescribed antimicrobial for the treatment of a chronic dento-alveolar infection was amoxicillin, typically prescribed for 5 days duration (66.3\%), or 7 days (30.5\%), a lower dose of $250 \mathrm{mg}$ of amoxicillin recorded on $16.7 \%$ of prescriptions. Where metronidazole was prescribed, it was usually for 5 days $(60.9 \%)$ or 7 days, (34.8\%) duration.

\section{Dry socket}

The management of dry socket involves local measures; with antimicrobials not routinely recommended. Practitioners reported prescribing for this indication with $7.7 \%$ of all prescriptions recorded for this indication. The most frequently prescribed antimicrobial for the treatment of a dry socket was metronidazole, typically prescribed for 5 days duration (59.4\%) or 7 days (24.6\%). Where amoxicillin was prescribed, it was usually for 5 days $(76.2 \%)$ or 7 days $(20.6 \%)$ duration.

\section{Discussion}

This antimicrobial audit has provided prospective data on the prescribing of antimicrobials by 275 dentists in the North East of England and Cumbria. The majority of the respondents were general dental practitioners and trained in the UK.

Amoxicillin was the most frequently prescribed antimicrobial, followed by metronidazole and then erythromycin. This pattern of prescribing is confirmed by the NHS Business Services Authority prescription data, the antimicrobial prescriptions dispensed by community pharmacies during October 2016 closely matches those reported in this audit [11]. Antibiotics such as co-amoxiclav, cephalosporins, clindamycin and quinolones, which do not appear on FGCP guidelines were prescribed infrequently, by participants in this audit.

The most common indication for antimicrobial prescribing was for the treatment of an acute dento-alveolar infection. Other common indications included pericoronitis, periodontal abscess, dry socket and chronic dento-alveolar infections. For conditions such as dry socket, routine use of antimicrobials is not recommended and therefore indicates a potential need for further education of practitioners.

For the treatment of acute-dento alveolar infections FGDP guidelines recommend that antimicrobials should only be prescribed as adjuncts to treatment and there should be evidence of systemic spread, pyrexia and local lymph gland involvement [3]. Results from this audit suggest that evidence of systemic spread, such a pyrexia or local lymph glad involvement were frequently absent when prescribing antimicrobials. This finding is similar to a recent study performed in Wales, where only $37.2 \%$ of patients prescribed antimicrobials had signs of spreading infection or systemic involvement [9].

The patient's condition was the key determining factor in practitioners prescribing antimicrobials; however, patient expectations were also frequent considerations in treatment choice. Work has been carried out in primary care medical practices to help educate patients about the unnecessary use of antimicrobials and there is evidence that public campaigns can potentially contribute to more careful use of antimicrobials [12]; this could be seen as a tool for reducing expectations in relation to dental prescribing.

Other reasons such as patient preference, time pressure and a lack of co-operation with other treatment options by patients were also commonly reported; these findings are similar to those from a recent study in Wales [13]. These are considerations for prescribers and further work to explore these factors using qualitative methods, would be beneficial in gaining a deeper appreciation of the reasons why practitioners prescribe antimicrobials, as well as what informs their choice of antimicrobial.

Following competition of the audit the dental antimicrobial stewardship (AMS) toolkit was published [5], by the Dental Subgroup of Public Health England's English surveillance programme for antimicrobial utilisation and resistance (ESPAUR), FGDP and British Dental Association (BDA). The toolkit produced provided an audit tool for antimicrobial prescribing and patient focused information such as information leaflets and posters [14].

\section{Limitations}

The audit was voluntary and reflected in the low response rate, therefore, those participating may be the practitioners who are most engaged in CPD and prescribing education. Therefore, those practitioners who were less likely to be up to date with current prescribing trends, were less likely to have been captured during this process. The audit was based on self-reports of the assessment of patient presentations and symptoms and also required dental practitioners to record data on antimicrobials that 
they prescribed over the audit period, rather than reporting retrospective data, as such the potential for practitioners to alter their prescribing habits (or checking the guidelines) needs to be taken into consideration.

\section{Recommendations}

Based on our results, we make several recommendations:

- Health Education England and the Local Dental Networks should highlight current guidance in relation to the local management of dental infections to practitioners, such as mail out of FGDP guidance to practices.

- Educational opportunities for practitioners, focusing on the recommended indications, duration of therapy and doses for antimicrobial prescriptions should be explored, for example, dissemination of guidance or CPD sessions.

- Opportunities to educate patients on antimicrobial use in dentistry should be reviewed. The posters produced as part of the AMS toolkit and patient information leaflet should be displayed in dental practices.

- Further research building on the existing literature exploring the underlying factors that lead to the prescribing of antimicrobials by dental practitioners should be considered. This could be achieved through qualitative research, utilising interviews or focus groups with dental practitioners.

\section{Conclusion}

This antimicrobial audit has shown that in most cases, when an antimicrobial is prescribed, practitioners are using the correct choice of agents and usually prescribing these at the correct dose. However, we did find some evidence of suboptimal prescribing practices which indicate that there may be a need for further support and/or training for prescribers. Underlying reasons for antimicrobial prescribing have been identified, but a deeper understanding is required to explore this fully and identify barriers or facilitators to improve clinical practice. The audit has identified training needs across the region and the development of CPD sessions on appropriate prescribing. The ESPAUR toolkit published subsequently to our audit provides an audit tool for practitioners.

\section{Additional files}

Additional file 1: Audit tool. Description - Audit tool used to collect data. (DOCX 263 kb)

Additional file 2: Table showing number and description of antimicrobial prescriptions from dental practices dispensed by pharmacies with contracts in the North East and Cumbria. Description Data provided by NHS BSA. (DOCX $16 \mathrm{~kb})$

\section{Abbreviations}

AMS: Dental Antimicrobial Stewardship; BDA: British Dental Association; BSA: Business Service Authority; CPD: Continued Professional Development; DDD: Defined Daily Dose; ESPAUR: English Surveillance Programme for Antimicrobial Utilisation and Resistance; FGDP: Faculty of General Dental Practitioners UK; GPT: General Professional Training; NHS: National Health Service; NICE: UK National Institute for Health and Care Excellence;

VT: Vocational Training

\section{Acknowledgements}

Not applicable.

\section{Funding}

This research was funded by a grant from NHS England. The funder assisted with the distribution of audit material, but had no involvement in the design, analysis, interpretation of data and preparation of the manuscript.

\section{Availability of data and materials}

The datasets used and/or analysed during the current study are available from the corresponding author on reasonable request.

\section{Authors contribution}

$A S, J L, T R, D L$ and $L B$ designed the study. $A O$ analysed the data which was checked and verified by $A S$ and $J$. AS prepared the manuscript which was reviewed and edited by $J, D L, T R, L B$, and $A O$. All authors have read and approved the final manuscript.

\section{Ethics approval and consent to participate}

The project was approved by the University of Sunderland Research Ethics Committee. Written consent to participate was not applicable.

\section{Consent for publication}

Not applicable.

\section{Competing interests}

The authors declare that they have no competing interests

\section{Publisher's Note}

Springer Nature remains neutral with regard to jurisdictional claims in published maps and institutional affiliations.

\section{Author details}

${ }^{1}$ Faculty of Health Sciences and Wellbeing, Sciences Complex, City Campus, Chester Road, University of Sunderland, Sunderland SR1 3SD, UK. ${ }^{2}$ PHE North East, Newcastle Upon Tyne, UK. ${ }^{3}$ Local Dental Network, Tees, Durham, Darlington, UK. ${ }^{4}$ Health Education North EastNewcastle Dental Hospital, Newcastle upon Tyne, UK.

Received: 28 February 2018 Accepted: 27 November 2018

Published online: 10 December 2018

\section{References}

1. World Health Organisation, 2014, Antimicrobial Resistance - Global Report on Surveillance 2014 http://apps.who.int/iris/bitstream/10665/112642/1/ 9789241564748_eng.pdf. Accessed Mar 2017.

2. National Institute for Clinical Excellence, 2015, Antimicrobial stewardship: systems and processes for effective antimicrobial medicine use. https:// www.nice.org.uk/guidance/ng15. Accessed Mar 2017.

3. The Faculty of General Dental Practitioners UK, 2014, Antimicrobial prescribing for general Dental practitioners, 2nd edition.

4. Health and Social Care Information Centre 2015, Prescribing by Dentists England 2014.

5. Public Health England, 2015, English surveillance programme for antimicrobial utilisation and resistance (ESPAUR) 2010-2015.

6. Jensen J, Bjerrum L, Boel J, Jarløv JO, Arpi M. Parents' socioeconomic factors related to high antibiotic prescribing in primary health care among children aged 0-6 years in the Capital Region of Denmark. Scand J Prim Health Care. 2016;34(3):274-81. https://doi.org/10.1080/02813432.2016.1207145.

7. Palmer NAO, Dailey YM, Martin MV. Can audit improve antimicrobial prescribing in general dental practice? Br Dent J. 2001;191:253. 
8. Chate RAC, White $S$, Hale LRO, et al. The impact of clinical audit on antimicrobial prescribing in general dental practice. Br Dent J. 2006;201: 635-41.

9. Cope AL, Barnes E, Howells EP, et al. Antimicrobial prescribing by dentists in Wales, UK: findings of the first cycle of a clinical audit. Br Dent J. 2016;221: 25-30.

10. British Dental Association. DFT and Vocational Training 2017. Online information available at https://www.bda.org/dentists/education/vocationaltraining. Accessed May 2017.

11. Bird L, Landes T, Robson A, Sturrock A, Ling J. Higher antibiotic prescribing propensity of dentists in deprived areas and those with greater access to care in the north east and Cumbria, UK. Br Dent J. 2018;225:517-24.

12. Hunter B, Goossens H, Verheij T, et al. Characteristics and outcomes of public campaigns aimed at improving the use of antimicrobials in outpatients in high-income countries. Lancet Infect Dis. 2010;10(1):17-31.

13. Cope AL, Francis NA, Wood F, Chestnutt IG. Antibiotic prescribing in UK general dental practice: a cross-sectional study. Community Dent Oral Epidemiol 2016; 44: 145-153. @ 2015 John Wiley \& Sons A/S. Published by John Wiley \& Sons Ltd.

14. Public Health England. Dental Antimicrobial stewardship:toolkit. 2016. https://www.gov.uk/guidance/dental-antimicrobial-stewardship-toolkit. Accessed Nov 2017

Ready to submit your research? Choose BMC and benefit from:

- fast, convenient online submission

- thorough peer review by experienced researchers in your field

- rapid publication on acceptance

- support for research data, including large and complex data types

- gold Open Access which fosters wider collaboration and increased citations

- maximum visibility for your research: over $100 \mathrm{M}$ website views per year

At BMC, research is always in progress.

Learn more biomedcentral.com/submissions 